\title{
Diagnosis of basal cell carcinoma by infrared spectroscopy of whole blood samples applying soft independent modeling class analogy
}

Mohammadreza Khanmohammadi • Razieh Nasiri •

Keyvan Ghasemi · Simin Samani •

Amir Bagheri Garmarudi

Published online: 24 August 2007

(C) Springer-Verlag 2007

\section{Erratum to: J Cancer Res Clin Oncol}

DOI 10.1007/s00432-007-0286-x

The original version of this article unfortunately contained a mistake. In the seventh sentence (14th line) of the section "Sample preparation and material", the wrong number of male patients was given. In fact, there were 43 males (not 53).

The online version of the original article can be found under doi:10.1007/s00432-007-0286-x.

M. Khanmohammadi $(\bowtie) \cdot$ R. Nasiri · K. Ghasemi ·

A. Bagheri Garmarudi

Department of Chemistry, Faculty of Science,

Imam Khomeini International University,

P.O. Box 288, Qazvin, Iran

e-mail:mrkhanmohammadi@gmail.com

S. Samani

School of Medicine,

Qazvin University of Medical Sciences,

Qazvin, Iran 